\title{
Association entre un taux indétectable de bicarbonate sérique et une pancréatite due à une hypertriglycéridémie
}

\author{
Nicole L. Veltri MD, Michael Chan PhD, Sara Awad MBBS
}

Citation : CMAJ 2020 October 13;192:E1206-9. doi : 10.1503/cmaj.200237-f

Voir la version anglaise de l'article ici : www.cmaj.ca/lookup/doi/10.1503/cmaj.200237

$\mathbf{U}$

ne femme de 48 ans s'est présentée au service d'urgence en raison de nausées, de vomissements et de douleur épigastrique irradiant dans le dos depuis 12 heures. Le mouvement et l'inspiration aggravaient la douleur, tandis que le fait de se pencher vers l'avant la diminuait. La patiente n'avait constaté aucun changement dans ses habitudes intestinales et ne présentait pas de polydipsie ni de polyurie. Ses antécédents médicaux comprenaient un diabète de type 2 mal contrôlé, diagnostiqué 3 ans plus tôt (hémoglobine glyquée $\left[\mathrm{HbA}_{1 \mathrm{c}}\right.$ ] 8,9\% [plage normale 4,0\%-6,0\%]), ainsi qu'une cholécystectomie. Elle prenait $1000 \mathrm{mg}$ de metformine 2 fois par jour.

La patiente consommait 25 paquets de cigarettes par année et fumait de la marijuana quotidiennement. Au cours des 6 mois précédents, sa consommation d'alcool avait augmenté pour atteindre 8 à 10 verres de spiritueux (1,5 once) par semaine. Elle disait ne pas avoir bu d'alcool dans la semaine précédant son arrivée à l'urgence, et elle mangeait de la malbouffe tous les jours. Parmi ses antécédents familiaux se trouvaient de l'hypercholestérolémie et du diabète de type 1 et de type 2, mais aucune maladie cardiaque prématurée.

À l'examen, la patiente était tachycarde (111 battements par minute), normotendue et afébrile, et elle ne présentait pas de respiration de Kussmaul. Même si sa pression veineuse jugulaire était de $7 \mathrm{~cm}$ de $\mathrm{H}_{2} \mathrm{O}$, ses membranes muqueuses étaient sèches; il a été déterminé qu'elle présentait une hypovolémie clinique. Une sensibilité gastrique a été constatée à l'examen abdominal. Aucun xanthome éruptif n'était visible.

Des examens de laboratoire ont révélé une hyperglycémie (glucose sanguin 16,7 mmol/L [plage normale 4-7 mmol/L]) et une hyponatrémie (sodium sérique $129 \mathrm{mmol} / \mathrm{L}$ [plage normale 133-145 mmol/L]; encadré 1 ). Le taux de sodium corrigé en fonction de l'hyperglycémie était de $132 \mathrm{mmol} / \mathrm{L}$. Le taux de bicarbonate n'était pas détectable à la mesure des électrolytes $\left(\mathrm{CO}_{2}\right.$ total), mais était normal à la gazométrie du sang artériel et veineux $\left(\mathrm{HCO}_{3}^{-}\right)$, avec des valeurs de $23 \mathrm{mmol} / \mathrm{L}$ et de $20 \mathrm{mmol} / \mathrm{L}$, respectivement. Le calcul manuel du trou anio-

\section{POINTS CLÉS}

- L'hypertriglycéridémie peut être à l'origine d'un écart important entre le taux de bicarbonate mesuré à l'analyse sérique des électrolytes $\left(\mathrm{CO}_{2}\right.$ total) et le taux de bicarbonate calculé à la gazométrie $\left(\mathrm{HCO}_{3}\right)$.

- L’hypertriglycéridémie peut interférer avec les analyses en laboratoire; le taux de $\mathrm{HCO}_{3}$ obtenu à la gazométrie est donc une valeur plus fiable et plus précise que le taux de $\mathrm{CO}_{2}$ total pour l'évaluation de l'équilibre acido-basique.

- Les patients au diabète mal contrôlé qui présentent une pancréatite due à une hypertriglycéridémie pourraient avoir un tableau clinique semblable à celui des patients qui présentent une acidocétose diabétique.

nique à partir d'un taux de $\mathrm{CO}_{2}$ total de $0-5 \mathrm{mmol} / \mathrm{L}$ a donné un résultat de 22-27 mmol/L (plage normale 10-14 mmol/L). La patiente présentait un taux légèrement élevé de lactate et de $\beta$-hydroxybutyrate, ainsi qu'un trou osmotique élevé $(25 \mathrm{mmol} / \mathrm{kg}$ [valeur normale $<10 \mathrm{mmol} / \mathrm{kg}$ ]). Son taux de lipase était haut ( $318 \mathrm{U} / \mathrm{L}$ [valeur normale $<79 \mathrm{U} / \mathrm{L}$ ]), et ses taux sériques d'alcools volatils, de salicylates, d'éthanol et d'acétaminophène étaient indétectables. La gazométrie a été réalisée avec un analyseur GEM 4000, la mesure de l'osmolarité sérique, avec un micro-osmomètre d'Advanced Instruments (modèle 3320), et la mesure de l' $\mathrm{HbA}_{1 \mathrm{c}}$, avec un analyseur VARIANT II. Les autres tests biochimiques ont été effectués au moyen du système ARCHITECT c16000 d'Abbott. Une tomodensitométrie de l'abdomen et du bassin a fait état de résultats concordant avec une pancréatite diffuse accompagnée de changements inflammatoires péripancréatiques, de liquides allant jusque dans le rétropéritoine et le quadrant supérieur droit, et d'un épaississement segmentaire dans l'angle droit du côlon.

La présentation clinique de la patiente et son taux élevé de lipase laissaient croire à une pancréatite aiguë. Cependant, les perturbations métaboliques étaient déroutantes, considérant 
Encadré 1 : Résultats des analyses de laboratoire chez une patiente de 48 ans présentant une pancréatite due à une hypertriglycéridémie

\begin{tabular}{|c|c|c|c|c|}
\hline Paramètres & $\begin{array}{l}\text { Plage normale chez } \\
\text { l'adulte }\end{array}$ & $\begin{array}{l}\text { À l'admission } \\
\text { (jour } 0,2 \text { heures) }\end{array}$ & $\begin{array}{l}\text { Avant l'aphérèse } \\
\text { (jour 1) }\end{array}$ & $\begin{array}{l}\text { Au congé } \\
\text { (jour } 5 \text { ) }\end{array}$ \\
\hline $\mathrm{Na}(\mathrm{mmol} / \mathrm{L})$ & $133-145$ & 129 & 134 & 136 \\
\hline Na corrigé en fonction de l'hyperglycémie (mmol/L)* & $133-145$ & 132 & 136 & 137 \\
\hline $\begin{array}{l}\text { Na corrigé en fonction de l'hyperlipidémie (valeur } \\
\text { théorique; } \mathrm{mmol} / \mathrm{L}) \dagger\end{array}$ & $133-145$ & 134 & N.D. $\ddagger$ & 137 \\
\hline $\mathrm{K}(\mathrm{mmol} / \mathrm{L})$ & $3,7-5,3$ & 3,5 & 2,9 & 3,6 \\
\hline $\mathrm{Cl}(\mathrm{mmol} / \mathrm{L})$ & $97-110$ & 102 & 105 & 102 \\
\hline $\mathrm{CO}_{2}$ total $(\mathrm{mmol} / \mathrm{L})$ & $19-27$ & $<5$ & 16 & 23 \\
\hline Trou anionique (mmol/L) & $8-12$ & N.D. & 13 & 11 \\
\hline Créatinine (mmol/L) & $0-110$ & 33 & 22 & 35 \\
\hline Glucose sanguin, (mmol/L) & $3,5-11,1$ & 16,7 & 13,1 & 9,5 \\
\hline Albumine (g/L) & $35-55$ & 28 & & 22 \\
\hline Calcium (mmol/L) & $2,25-2,80$ & 2,00 & 1,65 & 2,12 \\
\hline Lipase (U/L) & $0-79$ & 318 & & \\
\hline Lactate (mmol/L) & $0,5-2,2$ & 2,6 & 3,1 & 1,3 \\
\hline Osmolalité (mmol/kg) & $281-297$ & 302 & 292 & \\
\hline Trou osmolaire (mmol/kg) & & 25,1 & & \\
\hline \multicolumn{5}{|l|}{ Gazométrie veineuse } \\
\hline $\mathrm{pH}$ & $7,32-7,43$ & 7,33 & 7,49 & 7,35 \\
\hline $\mathrm{pCO}_{2}(\mathrm{mmHg})$ & $40-50$ & 44 & 32 & 45 \\
\hline $\mathrm{HCO}_{3}(\mathrm{mmol} / \mathrm{L})$ & $22-29$ & 23 & 24 & 25 \\
\hline \multicolumn{5}{|l|}{ Gazométrie artérielle } \\
\hline pH & $7,35-7,45$ & 7,30 & & \\
\hline $\mathrm{pCO}_{2}(\mathrm{~mm} \mathrm{Hg})$ & $35-45$ & 40 & & \\
\hline $\mathrm{pO}_{2}(\mathrm{~mm} \mathrm{Hg})$ & $80-100$ & 56 & & \\
\hline $\mathrm{pHCO}_{3}(\mathrm{mmol} / \mathrm{L})$ & $21-27$ & 20 & & \\
\hline Triglycérides (mmol/L) & $0-1,70$ & 36,78 & 23,68 & 2,33 \\
\hline Cholestérol (mmol/L) & $0-5,20$ & 16,00 & & 3,57 \\
\hline Cholestérol HDL (mmol/L) & $1,20-5,00$ & 0,55 & & 0,23 \\
\hline$\beta$-hydroxybutyrate (mmol/L) & $0-0,37$ & 0,54 & & $<0,20$ \\
\hline $\mathrm{HbA}_{1 c}(\%)$ & $4,0-6,0$ & & & 8,9 \\
\hline $\mathrm{TSH},(\mathrm{mUl} / \mathrm{L})$ & $0,40-4,50$ & & 0,76 & \\
\hline
\end{tabular}

que le taux de $\mathrm{CO}_{2}$ total était indétectable à l'analyse des électrolytes sériques et que celui de $\mathrm{HCO}_{3}{ }^{-}$était normal à la gazométrie. Même si le tableau clinique et le $\mathrm{pH}$ concordaient davantage avec une valeur de $\mathrm{HCO}_{3}{ }^{-}$normale, nous avons présumé que le taux de $\mathrm{CO}_{2}$ total était le plus fiable pour la prise de décisions (le risque de détérioration clinique de la patiente aurait été plus élevé si nous avions présumé à tort que le taux de $\mathrm{HCO}_{3}{ }^{-}$était le plus fiable).
Le diagnostic différentiel de l'acidose métabolique à trou anionique élevé comprenait l'acidocétose diabétique en contexte de diabète de type 1 plutôt que de type 2, et l'acidémie lactique due à une déshydratation, à un sepsis ou à la prise de metformine. Comme la patiente présentait une hyperglycémie ainsi qu'un trou anionique et un taux de cétones élevés, et comme nous n'avions aucune autre explication pour ces résultats, nous 
l'avons hospitalisée à l'unité de soins intermédiaires pour une pancréatite aiguë et une acidocétose diabétique.

Après avoir reçu $2 \mathrm{~L}$ de solution physiologique salée par voie intraveineuse, la patiente présentait un taux de sodium stable à $130 \mathrm{mmol} / \mathrm{L}$ et un trou anionique de 21-26 mmol/L. La correction rapide de son taux de sodium n'était pas une préoccupation, l'hyponatrémie observée étant principalement une pseudohyponatrémie. Le traitement de la patiente comprenait l'administration intraveineuse de $125-250 \mathrm{~mL}$ de solution physiologique salée par heure et de 4 unités d'insuline par heure, conformément au protocole de traitement de l'acidocétose diabétique de notre établissement. Étant donné nos préoccupations relatives au taux indétectable de $\mathrm{CO}_{2}$ total, nous avons administré une perfusion de bicarbonate de sodium à raison de 150-200 mL par heure, même si le pH était normal; cette partie du traitement ne faisait pas partie du protocole ${ }^{1}$. Le technicien de laboratoire nous a avisés que l'échantillon sanguin de la patiente était lipémique, et le bilan lipidique a mis en évidence une hypertriglycéridémie grave $(36,78 \mathrm{mmol} / \mathrm{L}$ [plage normale $0-1,70 \mathrm{mmol} / \mathrm{L}])$.

Un taux très faible de lipoprotéines de haute densité (HDL), un taux élevé de cholestérol et un taux considérablement élevé de triglycérides (encadré 1) laissaient croire que l'hypertriglycéridémie était à l'origine de la pancréatite. Nous avons demandé une consultation en endocrinologie. Nous nous sommes basés sur la valeur calculée du $\mathrm{HCO}_{3}{ }^{-}$, qui était normale, et le $\mathrm{pH}$ pour diagnostiquer une hyperglycémie découlant d'un dysfonctionnement des cellules $\beta$ occasionné par une pancréatite aiguë plutôt que d'une acidocétose diabétique. Les ressemblances entre la pancréatite due à une hypertriglycéridémie et l'acidocétose diabétique comprennent les douleurs abdominales, les nausées, les vomissements, la déshydratation, la tachycardie, l'hyperglycémie, la pseudohyponatrémie, un faible taux de bicarbonate de sodium et un trou anionique important. Nous avons mis fin au protocole de traitement de l'acidocétose diabétique et avons entrepris un traitement du diabète par injections sous-cutanées d'insuline.

Malgré l'amélioration de son taux de triglycérides $(23,68 \mathrm{mmol} / \mathrm{L})$ après un repos intestinal et l'administration intraveineuse de liquides et d'insuline, la patiente n'était toujours pas bien sur le plan clinique, ayant encore des douleurs abdominales et des vomissements. Comme elle a développé une hypocalcémie de $1,65 \mathrm{mmol} / \mathrm{L}$ (encadré 1 ), nous avons procédé à une aphérèse pour accélérer l'abaissement de son taux de triglycérides. Nous lui avons installé un cathéter d'hémodialyse, après quoi elle a subi un cycle d'aphérèse qui a permis une amélioration marquée. Son taux de triglycérides a baissé $(3,45 \mathrm{mmol} / \mathrm{L})$, tout comme son taux de $\mathrm{CO}_{2}$ total $(19 \mathrm{mmol} / \mathrm{L})$. La patiente a ensuite commencé à prendre du fénofibrate (200 mg/jour), de la rosuvastatine $(20 \mathrm{mg} / \mathrm{j})$ et de l'huile de poisson riche en oméga-3 (2-3 g/j). Elle a également recommencé à prendre de la metformine, à raison de $1000 \mathrm{mg}$ 2 fois par jour.

La patiente a obtenu son congé après 5 jours, et elle devait s'administrer 12 unités d'insuline glargine chaque soir au coucher. Au moment du congé, son taux de triglycérides était de 2,33 $\mathrm{mmol} / \mathrm{L}$, et son taux de $\mathrm{CO}_{2}$ total était normal, à $23 \mathrm{mmol} / \mathrm{L}$. Nous n'avons pas fait de tests génétiques pour trouver les causes primaires de son hypertriglycéridémie, puisque son alimentation, sa consommation d'alcool et son diabète étaient des facteurs secondaires contributifs sur lesquels il fallait travailler. Son très faible taux sérique de HDL était non spécifique, et était possiblement lié au syndrome métabolique, à l'inflammation aiguë ou à l'hyperlipidémie primaire. Un suivi avec son médecin de famille et l'endocrinologue a été prévu.

\section{Discussion}

\section{Facteurs de risque d'une pancréatite due à une hypertriglycéridémie}

L'hypertriglycéridémie résulte d'une combinaison de causes primaires (génétiques) et secondaires. Les causes secondaires comprennent un syndrome métabolique, un diabète mal contrôlé (généralement de type 2 ) en raison d'une résistance à l'insuline, et une consommation excessive d'alcool'2.

Bien que l'hypertriglycéridémie, même légère ou modérée, soit un facteur de risque de maladie cardiovasculaire, elle n'est pas un facteur de risque important de pancréatite, à moins d'être grave (>11,2 mmol/L), ce qui est rare en cas d'hypertriglycéridémie isolée acquise ${ }^{2}$. Ainsi, chez les patients atteints d'une pancréatite due à une hypertriglycéridémie, les causes sous-jacentes des troubles du métabolisme des lipides doivent être prises en considération ${ }^{2}$.

Les patients qui développent une pancréatite due à une hypertriglycéridémie ont tendance à présenter une élévation aiguë ou chronique du taux sérique de triglycérides attribuable à des facteurs secondaires non maîtrisés, comme un diabète sucré, une grossesse ou une consommation importante d'alcool ${ }^{3-5}$. Le diabète sucré est à l'origine de $62 \%-9 \%$ des cas de pancréatite due à une hypertriglycéridémie ${ }^{3,4}$.

\section{Présentation clinique et diagnostic}

La pancréatite due à une hypertriglycéridémie a une présentation clinique semblable à celle d'une pancréatite ayant une autre étiologie; cependant, elle s'accompagne d'un risque accru de défaillance d'organe et d'hospitalisation aux soins intensifs ${ }^{5,6}$. Le diagnostic de pancréatite aiguë selon la classification révisée d'Atlanta nécessite au moins 2 des critères suivants : douleurs abdominales caractéristiques d'une pancréatite aiguë, taux sérique de lipase ou d'amylase au moins 3 fois supérieur à la normale, et signes de pancréatite aiguë visibles aux examens d'imagerie.

La pancréatite due à une hypertriglycéridémie représente $2 \%-10 \%$ des cas de pancréatite aiguë. On la laisse souvent de côté, lui préférant des causes plus courantes, comme les calculs biliaires et l'alcool ${ }^{5}$. Or, comme mentionné plus tôt, la consommation excessive d'alcool est un facteur de risque de pancréatite récurrente due à une hypertriglycéridémie (rapport de risque 3,40 [intervalle de confiance à $95 \% 1,37-8,42])^{4}$. Un autre facteur de confusion dans le diagnostic est l'épiphénomène d'élévation légère ou modérée du taux sérique de triglycérides 
observé dans $33 \%$ des pancréatites aiguës de toutes causes ${ }^{7}$. Cela dit, les données observationnelles disponibles indiquent que la pancréatite due à une hypertriglycéridémie est rare en présence d'un taux sérique de triglycérides supérieur à $20 \mathrm{mmol} / \mathrm{L}(<5 \% \text { des cas })^{2,5}$.

\section{Résultats de laboratoire}

Les résultats de laboratoire en cas de pancréatite due à une hypertriglycéridémie ressemblent à ceux observés en cas de pancréatite d'autre étiologie, mais plusieurs différences importantes sont présentes. La pseudohypobicarbonatémie, définie comme un écart entre les taux de $\mathrm{HCO}_{3}{ }^{-}$et de $\mathrm{CO}_{2}$ total principalement attribuable à une interférence en laboratoire, est d'ailleurs bien connue (encadré 2 ) $^{8,9}$. Notre établissement se sert de méthodes enzymatiques spectrophotométriques pour mesurer les électrolytes. Dans ce type de tests, les grandes particules comme les chylomicrons et les lipoprotéines de très basse densité absorbent la lumière et provoquent des interférences durant l'analyse, ce qui donne un taux faussement bas de $\mathrm{CO}_{2}$ total ${ }^{8,9}$. À l'opposé, dans la gazométrie sanguine, le taux de bicarbonate est calculé à partir du pH sanguin et de la pression partielle du $\mathrm{CO}_{2}$, qui ne sont pas sensibles à ces interférences.

Encadré 2 : Diagnostic différentiel de l'écart entre le taux mesuré de $\mathrm{CO}_{2}$ total et le taux calculé de $\mathrm{HCO}_{3}{ }^{10,13}$

\begin{tabular}{|lc|}
\hline Causes sous-jacentes & $\begin{array}{c}\text { Valeur } \\
\text { touchée }\end{array}$ \\
\hline Erreur de manipulation de l'échantillon & \\
\hline $\begin{array}{l}\text { Excédent d'héparine dans l'échantillon destiné à la } \\
\text { gazométrie }\end{array}$ & $\downarrow \mathrm{HCO}_{3}$ \\
\hline \begin{tabular}{l} 
Perte de l'anaérobie dans l'échantillon \\
\hline Erreur de calibrage ou imprécision de l'appareil
\end{tabular} & $\downarrow \mathrm{CO}_{2}$ total \\
\hline $\begin{array}{l}\text { Problèmes de santé } \\
\text { Hypertriglycéridémie }\end{array}$ & $\downarrow / \uparrow \mathrm{HCO}_{3}$ \\
\hline et $\mathrm{CO}_{2}$ \\
total
\end{tabular}

Une pseudohyponatrémie peut également être présente en cas d'hypertriglycéridémie. Notre hôpital se sert de techniques indirectes faisant appel à des électrodes sensibles à des ions spécifiques pour la mesure des électrolytes. Dans ces analyses, les échantillons sont dilués avant la mesure des électrolytes. Étant donné le déplacement de volume important observé lors d'une hyperlipidémie grave, il est possible que les résultats mettent faussement en évidence une faible concentration d'électrolytes, menant ainsi à une pseudohyponatrémie. Les échantillons lipémiques peuvent être traités par ultracentrifugation ou avec un réactif avant leur analyse ${ }^{8,10}$.

On a proposé une formule pour calculer le taux corrigé de sodium ${ }^{11}$ : taux corrigé $\left[\mathrm{Na}^{+}\right]=$taux mesuré $\left[\mathrm{Na}^{+}\right]+$(lipides totaux/10), formule dans laquelle les valeurs du [ $\left.\mathrm{Na}^{+}\right]$et des lipides totaux (cholestérol et triglycérides) sont exprimées en $\mathrm{mmol} / \mathrm{L}$.

Enfin, un trou anionique élevé a été signalé chez les patients qui présentent une hypertriglycéridémie grave et une pseudohyponatrémie; on croit qu'il est causé par une lipolyse produisant des ions d'hydrogène excessive, qui influence le titrage sérique du $\mathrm{HCO}_{3}^{-8,9}$. Il est crucial que les cliniciens connaissent les facteurs d'interférence pouvant survenir en laboratoire pour poser le bon diagnostic.

\section{Prise en charge}

Il n'existe aucune ligne directrice fondée sur des données probantes visant la prise en charge de la pancréatite due à une hypertriglycéridémie. Le traitement conservateur comprend le repos intestinal, l'administration de liquides et le soulagement de la douleur. La perfusion d'insuline a également été présentée comme une stratégie de traitement sûre et efficace chez les patients, qu'ils soient atteints de diabète ou non ${ }^{3,6}$. L'insuline active l'enzyme lipoprotéine lipase, ce qui augmente la dégradation des chylomicrons et abaisse le taux de triglycérides ${ }^{3,6}$.

L'héparine administrée par voie intraveineuse accroît aussi l'activité de la lipoprotéine lipase et peut être utilisée de façon synergique pour réduire le taux de triglycérides en cas de pancréatite grave $e^{3,6}$. L'administration intermittente de bolus est préférable à l'administration continue pour atténuer le risque de complications par saignement et d'hypertriglycéridémie secondaire à l'épuisement de la lipoprotéine lipase $e^{3,6}$.

L'aphérèse a été présentée comme une solution efficace pour soulager les symptômes associés à la réduction rapide des triglycérides et à la baisse de l'inflammation ${ }^{6}$. Cependant, des études récentes n'ont montré aucun avantage sur les issues cliniques et ont fait état de complications potentielles, comme une réaction allergique ou une infection liée à la perfusion ${ }^{3,6}$.

La prise en charge à long terme comprend des interventions non pharmacologiques (modification de l'alimentation, exercice et perte de poids) et pharmacologiques (fibrates, niacine et acides gras oméga-3, avec ou sans statines) $)^{2}$.

\section{Références}

1. Diabetes Canada Clinical Practice Guideline Expert Committee; Goguen J, Gilbert J. Diabetes Canada 2018 clinical practice guidelines: hyperglycemic emergencies in adults. Can J Diabetes 2018;42:S109-14.

2. Berglund L, Brunzell JD, Goldberg AC, et al.; Endocrine society. Evaluation and treatment of hypertriglyceridemia: an Endocrine Society clinical practice guideline. J Clin Endocrinol Metab 2012;97:2969-89.

3. Shemesh E, Zafrir B. Hypertriglyceridemia-related pancreatitis in patients with type 2 diabetes: links and risks. Diabetes Metab Syndr Obes 2019;12:2041-52.

4. Zafrir B, Saliba W, Jubran A, et al. Severe hypertriglyceridemia-related pancreatitis: characteristics and predictors of recurrence. Pancreas 2019;48: 182-6. 
5. de Pretis N, Amodio A, Frulloni L. Hypertriglyceridemic pancreatitis: epidemiology, pathophysiology and clinical management. United European Gastroenterol J 2018;6:649-55

6. Rawla P, Sunkara T, Thandra KC, et al. Hypertriglyceridemia-induced pancreatitis: updated review of current treatment and preventive strategies. Clin J Gastroenterol 2018;11:441-8.

7. Balachandra S, Virlos IT, King NKK, et al. Hyperlipidaemia and outcome in acute pancreatitis. Int J Clin Pract 2006;60:156-9.

8. Rifkin SI, Shaub B. Factitious hypobicarbonatemia associated with profound hyperlipidemia. Ren Fail 2014;36:1155-7.

9. Stein H. Spuriously low serum bicarbonate levels in patients with hyperlipidemia: a report of 4 cases. Am J Kidney Dis 2019;73:131-3.

10. Castro-Castro MJ, Candás-Estébanez B, Esteban-Salán M, et al.; Commission on Lipoprotein and Vascular Diseases, Sociedad Española de Química Clínica. Removing lipemia in serum/plasma samples: a multicenter study. Ann Lab Med 2018;38:518-23.

11. Dimeski G, Mollee P, Carter A. Effects of hyperlipidemia on plasma sodium, potassium, and chloride measurements by an indirect ion-selective electrode measuring system. Clin Chem 2006;52:155-6.

12. Cartier JL, Look DC, Dunn JP. Pseudohypobicarbonatemia in a patient presenting with suspected diabetic ketoacidosis. AACE Clin Case Rep 2018;4:e108-11.

13. Lazzouni I, Cornec E, Meskar A, et al. Pseudohypobicarbonatémie chez une patiente atteinte d'une gammapathie monoclonale à IgA kappa. Ann Biol Clin (Paris) 2014;72:599-601.

Intérêts concurrents : Aucun déclaré.

Cet article a été révisé par des pairs.

Les auteurs ont obtenu le consentement de la patiente.

Affiliations : Division de médecine interne générale (Veltri), Département de médecine, et départements de pathologie et de médecine moléculaire (Chan), et Division d'endocrinologie et de métabolisme (Awad), Département de médecine, Université Queen's, Kingston (Ontario).

Collaborateurs : Tous les auteurs ont contribué à l'élaboration et à la conception de l'étude, ont rédigé le manuscrit et en ont révisé de façon critique le contenu intellectuel important; ils ont donné leur approbation finale pour la version destinée à être publiée et assument l'entière responsabilité de tous les aspects du travail.

Correspondance : Nicole Veltri, 12nlv@queensu.ca 